\title{
On the scaling of the slip weakening rate of heterogeneous faults
}

\author{
H. Perfettini and M. Campillo \\ Laboratoire de Géophysique Interne et Tectonophysique, Grenoble, France
}

I. Ionescu

Laboratoire de Mathématiques, Université de Savoie, Le Bourget du Lac, France

Received 10 May 2002; revised 23 September 2002; accepted 21 March 2003; published 3 September 2003.

[1] We present an attempt to describe the scaling law of the slip weakening rate at the onset of instability using a two-dimensional fault model. A fault consists of a series of weak patches under slip weakening friction, separated by unbreakable barriers. A first group of faults contains an even distribution of patches of different scales conserving the same total slipping length, while a second group consists of various fractal Cantor sets. The global behavior of rupture is described by the exponential growth rate $\lambda$. For an infinite homogeneous fault, the coefficient $\lambda$ is governed by the weakening rate of the friction law. We estimate the weakening rate of each individual fault in an heterogeneous fault system such that the rate of exponential growth $\lambda$ of this fault network is identical to that of a single homogeneous fault. Using this homogenization procedure, we compute the weakening rate on the weak patches for faults with different scales of heterogeneity and a given $\lambda$. At large scales, the weakening rate is scale-independent, the initiation process on a long patch being similar to the case of an infinite fault. At small scales and for all the different geometries considered here, the weakening rate varies as $\alpha=\beta_{0}^{*} / a$, where $a$ is the scale or half length of each elementary fault and $\beta_{0}^{*} \simeq 1.158$. We discuss the physical implications of our results on the value of the slip weakening distance $D_{c}$ and give a possible explanation of the scale dependence of this parameter. INDEX TERMS: 7209 Seismology: Earthquake dynamics and mechanics; 7215 Seismology: Earthquake parameters; 7260 Seismology: Theory and modeling; KEYWORDS: effective friction law, scaling of frictional parameters, renormalization, heterogeneous faults, earthquake dynamics

Citation: Perfettini, H., M. Campillo, and I. Ionescu, On the scaling of the slip weakening rate of heterogeneous faults, J. Geophys. Res., 108(B9), 2410, doi:10.1029/2002JB001969, 2003.

\section{Introduction}

[2] Understanding the frictional behavior of a fault is a key issue of earthquake physics. One way to proceed is to extrapolate the frictional behavior of rocks inferred from laboratory rock experiments. Because of the complexity of a real fault network compared to a rock sample, extrapolating the laboratory-based frictional laws to the fault scale is far from being trivial. To illustrate that point, it is important to notice that the characteristic length $D_{c}$ of evolution of friction with ongoing slip is measured to be of the order of $10 \mu \mathrm{m}$ [Ohnaka, 1986] in laboratory experiments studying rock friction, while seismological evidences (e.g., kinematic inversions) seems to favor a $D_{c}$ of the order of a meter [Ide and Takeo, 1997; Olsen et al., 1997]. There are two possible explanations to this discrepancy:

[3] 1. The physics is different for a real fault than for a rock sample, for instance due to the presence fault gouge. For instance, [Chambon et al., 2002] have carried on a laboratory experiment in which they have studied the

Copyright 2003 by the American Geophysical Union. 0148-0227/03/2002JB001969\$09.00 frictional behavior of a gouge material, showing the existence of a slip weakening distance of the order of a meter. Therefore a metric $D_{c}$ (a value in agreement with the seismological estimates of this parameter) may be measured at the laboratory scale due, in the study of Chambon et al. [2002], to the presence of a granular medium.

[4] 2. The physics is essentially the same but the values of the frictional parameters are scale-dependent. Such would be the case if $D_{c}$ was an effective rather than a physical parameter (i.e., a material property). For example, if the measure of the parameter $D_{c}$ was strongly influenced by the interactions between microcracks in the fault zone, then the measurement of $D_{c}$ would be extremely scale-dependent. Assuming that the frictional behavior of a fault obeys a simple slip weakening law, we test point 2 by studying the scale dependence of the weakening rate $\alpha$, where $\alpha=$ $\Delta \tau /\left(\mu D_{c}\right)$ for a linear slip weakening law, where $\mu$ is the shear modulus and $\Delta \tau$ is the stress drop of the event.

\section{The Model}

[5] We assume that the fault network consists of elementary faults separated by unbreakable barriers, each of the 
elementary faults obeying a simple slip weakening law with the same weakening rate $\alpha$. The faults of half length $a$ are located in the $(x, z)$ plane at $y=0$, and slip $w(t, x, y)$ occurs in the $z$ direction which is also a direction of invariance. Each point of the volume surrounding the fault obeys the wave equation:

$$
\frac{\partial^{2} w}{\partial t^{2}}(t, x, y)=c^{2} \nabla^{2} w(t, x, y), \quad \forall y>0
$$

where we have limited our study to the upper half-space $(y>0)$. The boundary conditions are

$$
w(t, x, 0)=0
$$

in the barriers and

$$
a \frac{\partial w}{\partial y}(t, x, 0)=-\beta w(t, x, 0)
$$

on the elementary faults. Equation (2) imposes no slip outside of the faults, while each subfault obeys a linearized slip weakening law involving the nondimensional constant $\beta=a \alpha$ (see equation (3)). We prescribe that the slipping patches are on the verge of instability, and equation (3) is therefore valid during the initiation phase, i.e., for all $w(t, x, 0) \leq D_{c}$. Equations (1)-(3) are equivalent in the spectral domain to the eigenvalue problem:

$$
\begin{gathered}
\nabla^{2} \phi(x, y)=\lambda^{2} \phi(x, y), \quad \forall y>0, \\
\phi(x, 0)=0
\end{gathered}
$$

in the barriers, and

$$
a \partial_{y} \phi(x, 0)=-\beta \phi(x, 0)
$$

on the slipping patches, where $\varphi$ is the eigenfunction with eigenvalue $\lambda^{2}$. The largest eigenvalue, $\lambda_{0}^{2}(\beta)$, is positive for the unstable process studied here. The evolution of slip with time is controlled by the dominant term $w \propto \exp \left(c\left|\lambda_{0}\right| t\right)$. C. Dascalu and I. Ionescu (Spectral analysis of instabilities for the wave equation and earthquake initiation, submitted to SIAM Journal of Applied Mathematics, 2001, hereinafter referred to as Dascalu and Ionescu, submitted manuscript, 2001) have shown that $\lambda_{0}^{2}$ increases with $\beta$, and defined the inverse function $\beta_{0}=\beta(\lambda)$, i.e., $\lambda_{0}^{2}\left(\beta_{0}(\lambda)\right)=\lambda^{2}$. They also reduced the above eigenvalue problem to a hypersingular integral equation, and developed a semianalytical technique to compute the value of the parameter $\beta$ for given eigenvalue $\lambda_{0}^{2}$. The method of computation is based on a solution of the spectral problem solved with a boundary integral equation technique.

[6] Two end-member cases are particularly interesting to interpret our results. For a single and finite fault of half length $a$, Dascalu et al. [2000] found that the system is stable if $\beta<\beta_{0}^{*}$ but unstable if $\beta>\beta_{0}^{*}$, with $\beta_{0}^{*}=\beta_{0}(\lambda=0) \simeq$ 1.15777388. Recalling that $\beta=a \alpha$, this implies that faults smaller than $a_{c}$ are stable while an "earthquake" is expected on faults larger than $a_{c}$. The critical half length $a_{c}=\beta_{0}^{*} / \alpha$ is called the nucleation size and represents the smallest size of an instability. For an infinite fault, slip is always unstable but the characteristic size of the slipping zone is now $a_{c}^{\infty}=$ $\pi / \alpha$ [Campillo and Ionescu, 1997].
[7] We assume that at large scale, the fault network behaves as a single fault of half length $a_{0}$. The degree of complexity, i.e., the number of secondary faults that is resolved, decreases with increasing scale of observation. To build the fault network at smaller scale, a procedure or renormalization scheme is needed. We have used a large number of such schemes that we will briefly described here. The first type of sets are the ones labeled as $N=n_{0} R=n_{1}$, where $n_{0}$ and $n_{1}$ are two integers. It means that one goes from one scale to the immediately lower one by replacing each fault by $n_{0}$ daughter faults, each of them being $1 / n_{1}$ smaller than the mother fault. Such sets can be seen on Figure 1a $(N=2 R=4), 1 \mathrm{~b}(N=3 R=9)$ and $1 \mathrm{c}(N=2 R=3$ with voids $)$. One can also impose a renormalization procedure for the barriers as in the case $N=2 R=3$ of Figure 1d, where each barrier is replaced by two secondary barriers separated by a fault. In the second set, the proportion faults/barriers is held constant at all scales such as the case of $10 \%$ or $90 \%$ of faults of Figures 1e and 1f. The sets $N=2 R=4, N=3 R=9$, and $N=2 R=3$ with voids (also called the triadic Cantor set) are fractal sets with fractal dimension $D=0.5,0.5$, and 0.6309 , respectively [Korvin, 1992]. Those sets are of particular interest since it has been proposed that fault networks obey a fractal distribution [Scholz, 1990].

[8] Our study of the scaling of the weakening rate is based on the following approach. Let us assume a particular slip evolution characterized by the eigenvalue $\lambda$ that describes completely the collective temporal behavior of the actual network. We can associate this eigenvalue $\lambda$ with an effective weakening rate which, when applied to an homogeneous fault, produces a slip evolution similar in average to the one of the heterogeneous fault. The validity of this approach for the complete law has been demonstrated by Campillo et al. [2001] and Voisin et al. [2002]. Our purpose here is limited to the initial weakening rate but accounts for several changes of scale. Considering that in practice only the large-scale behavior can be deduced from seismological data, we fix a value of $\lambda_{0}$ and compute the weakening rate on the slipping patches that produces the eigenvalue $\lambda$ when the geometric complexity is described up to a scale $a$. We use values of $\lambda$ lower than 1 , this assumption being discussed in section 4 .

\section{Results}

[9] In the following calculations, we impose the instability to grow as $\lambda$ with $\lambda>0$, in order to consider cases far enough from the static stability boundary, i.e., $\lambda=0$. Figure 2 shows the eigenfunction or slip distribution $\phi_{0}$ associated with the eigenvalue $\lambda_{0}=0.01$ (associated with the most unstable mode) for the first 6 iterations of the renormalizations considering the $N=2 R=3$ scheme. We see that at all scales, the average slip distribution (or its envelop) remains qualitatively the same, and similar to the case of one single fault (case $N=1$ of Figure 2). This observation illustrates the existence of a spectral equivalence between the problem at different scales of heterogeneity.

[10] Using the numerical approach developed by Dascalu and Ionescu (submitted manuscript, 2001), we estimate the value $\beta_{0}(\lambda)$ at different stages of the renormalization procedures, i.e., for different half size $a$ of the elementary 
a) $\mathrm{N}=2, \mathrm{R}=4$

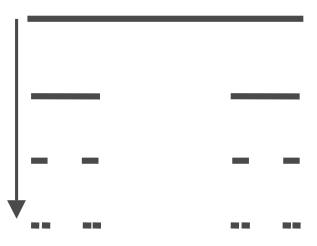

c) $\mathrm{N}=2, \mathrm{R}=3$ with voids

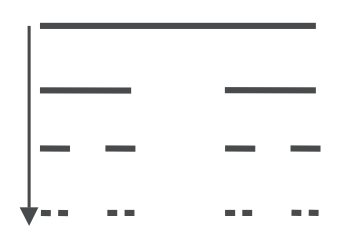

e) $10 \%$ of faults

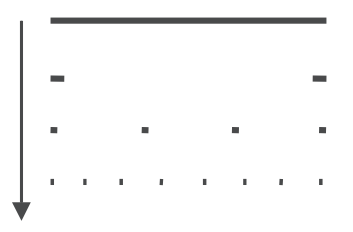

b) $\mathbf{N}=3, R=9$

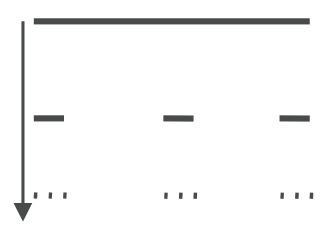

d) $\mathrm{N}=2, \mathrm{R}=3$

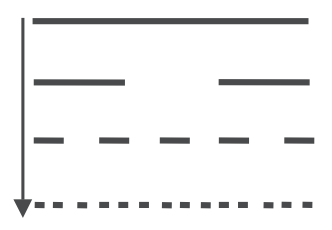

e) $90 \%$ of faults

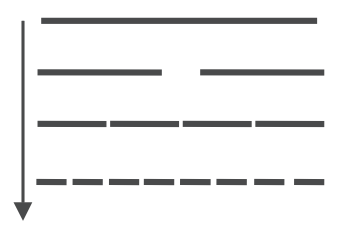

Figure 1. Renormalization schemes used in this study. At large scale, the fault system can be replaced by a single fault, while the underlying fault structure becomes more complex at smaller scales.

faults. For a given scale $a$, the weakening rate $\alpha=\beta_{0}(\lambda) / a$ is plotted on Figure 3 for the cases $\lambda_{0}=0$ and 0.1. This last value of $\lambda_{0}$ is arbitrary and was chosen in order to illustrate the two asymptotic regimes observed in this study: At large scale the weakening rate is constant while at smaller scale the scaling $\alpha \propto 1 / a$ applies. It is remarkable to see that such scaling seems independent of the renormalization scheme considered, and remains valid for fractal sets as well as for a periodic fault network.

[11] The nucleation half length $a_{c}$ is a natural cutoff length for an infinite fault. If an elementary finite fault is much larger than the nucleation length, i.e., $a \gg a_{c}$, then this fault may be treated as being infinite, since only a small fraction of the fault is slipping during the initiation process. As discussed by Campillo and Ionescu [1997], the most unstable mode for an infinite fault grows with time as $\lambda=\alpha$, leading to $\alpha=0.1 \mathrm{~m}^{-1}$ for our particular choice of $\lambda=0.1 \mathrm{~m}^{-1}$. This is indeed the asymptotic value that is observed in Figure 3 at large scales.

[12] After a sufficient number of iterations of the renormalization process, the size of the elementary fault is much smaller than the nucleation size, i.e., $a \ll a_{c}$. In that case, the finiteness of the elementary faults can no longer be neglected. After introducing the dimensionless variable $\hat{y}=y / a$, the boundary condition (3) yields to $\partial w(t, x, 0) /$ $\partial \hat{y}=-\beta w(t, x, 0)$, showing that the dynamics of the fault (described by the parameter $\lambda$ ) is only controlled by the parameter $\beta=a \alpha$ and possibly the number $n$ of subfaults (or

equivalently the scale $a$ ), i.e, $\lambda=\lambda(n, \beta)$. Nevertheless, for scales much smaller than the nucleation size $\left(a \ll a_{c}^{\infty}\right)$, the dependence of $\lambda$ upon the number of subfaults vanishes due to the self-similarity of the geometry we consider, leading to $\lambda=\lambda(\beta)$. Therefore one expects that at small scales the parameter $\beta$ only depends on the divergence rate $\lambda$, leading to the following dependence for the weakening rate: $\alpha=\beta(\lambda) / a$. As illustrated in Figure 3, our numerical simulations show that $\beta \simeq C$, where $C$ is a constant. Only the cases $\lambda=0$ and $\lambda=0.1$ are presented on Figure 3 for a sake of clarity, but we have verified these results over a wide range of $\lambda(0<\lambda<1)$. We have found that the value $\beta(\lambda=0) \simeq 1.15777388$ found by Dascalu et al. [2000] was a good estimate of our constant $C$. Therefore we assume in the rest of this work that $C \simeq 1.15777388$. This weak dependence of $\beta$ to variations of $\lambda$ for small $\lambda(\lambda<1)$ has been previously shown by Dascalu et al. [2000] considering a single finite fault. In other words, we consider here fault systems that are close to their limit of stability, a realistic assumption for actual faults.

[13] The fact that $\alpha$ decreases with $a$ can be understood by noting that large faults are always more unstable than small faults, since they are further away from the stability boundary defined by the nucleation size. Therefore, as the size (or scale) of the fault is increasing, a lower weakening rate is needed to lead to the same divergence rate $\lambda$.

[14] As previously noted, the two asymptotic regimes are defined below and above a cross over length equal to the nucleation half length $a_{c}^{\infty}=\pi / \alpha$ [Campillo and Ionescu, 1997]. It is represented by a continuous vertical line in Figure 3. For an infinite fault, the most unstable mode corresponds to $\alpha=\lambda$, and hence the crossover length $a_{c}^{\infty}$ is also given by $a_{c}^{\infty}=\pi / \lambda$.

\section{Discussion}

[15] We have studied the initiation of slip instability on a fault network with scale-dependent pattern. In order for the macroscopic coefficient of exponential growth with time to be constant at all scales, we have shown that the weakening rate $\alpha=\Delta \tau /\left(\mu D_{c}\right)$ has to be inversely proportional to the scale of observation, i.e., $\alpha=$ const $/ a$ with const $=\beta_{0}^{*}$, up to a scale of the order of the nucleation size $a_{c}^{\infty}$ of an infinite fault. The simple function

$$
\alpha=\max \left[\beta_{0}^{*} / a, \lambda\right],
$$

where $\max [x, y]$ is the maximum between $x$ and $y$, gives a rough estimate of the weakening rate $\alpha$. Our results were obtained considering regular fault networks. Since we have shown that those results were independent on the detailed pattern of the fault network, we expect them to be valid for nonregular (e.g., stochastic) fault networks, even though we have not tested such an assumption.

[16] A fundamental question is to know in which regime (i.e., the $\alpha \propto 1 / a$ or the $\alpha \simeq$ const regime) do natural faults evolve. Let us first suppose that the fault network of characteristic scale $a$ evolves in the large-scale regime for which $a \gg a_{c}^{\infty}$ and $\alpha=\lambda$. We start by estimating the weakening rate $\alpha$ using seismological observations. Taking $D_{c} \simeq 1 \mathrm{~m}$ and $\Delta \tau \simeq 1 \mathrm{MPa}$ [Ide and Takeo, 1997], and using $\alpha$ of the order of $\mu=30 \mathrm{GPa}$, we find that $\alpha \simeq 510^{-5} \mathrm{~m}^{-1}$, leading to a nucleation size of $a_{c}^{\infty}=\pi / \lambda \simeq 62.8 \mathrm{~km}$. This 

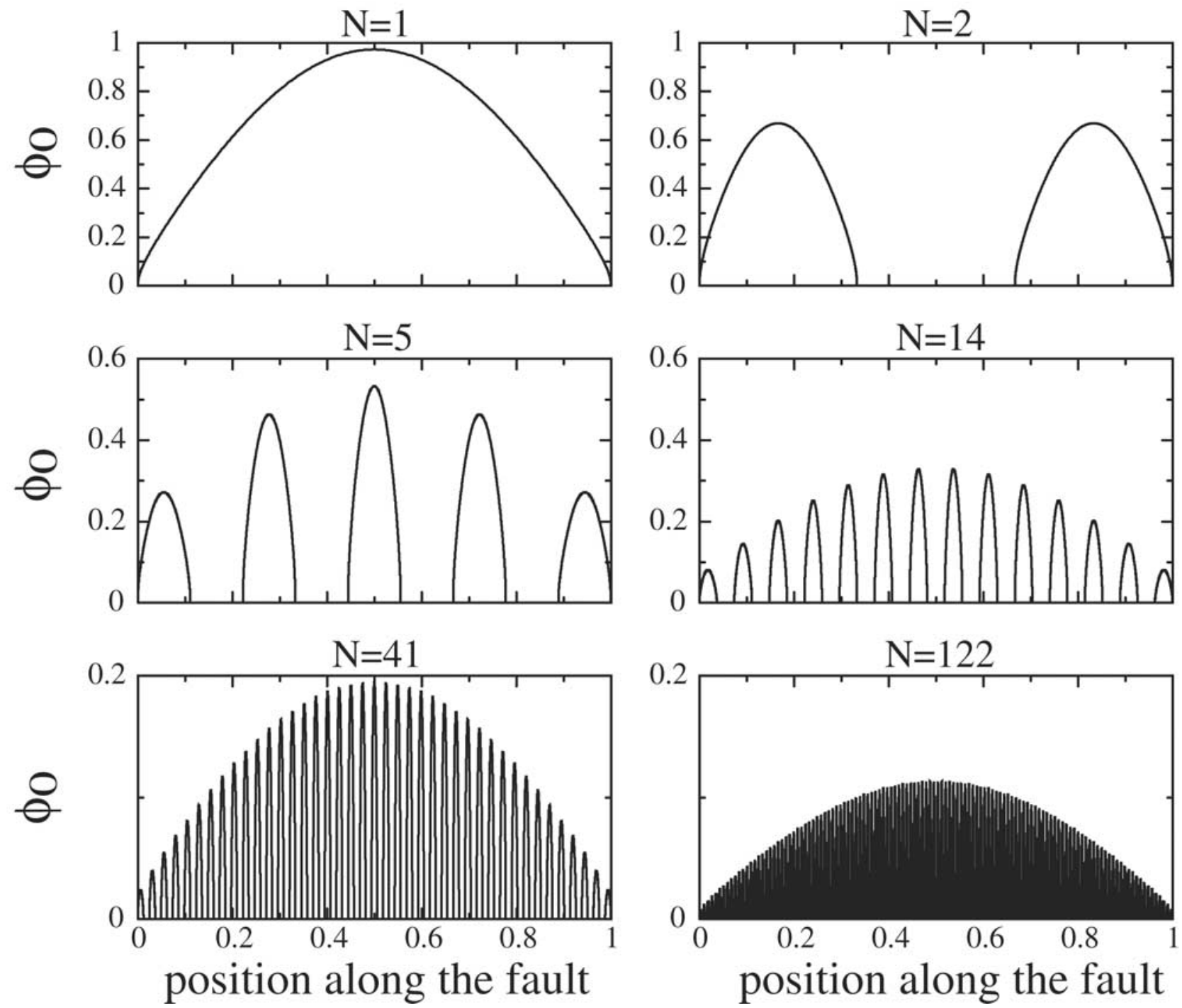

Figure 2. Slip distribution for various number of faults corresponding to different scales using the $N=2$ $R=3$ renormalization scheme with $\lambda=0.01$. For each step of the renormalization procedure, the integer $N$ represents the total number of secondary faults of the fault network. The position along the fault system is normalized by the total length of the system $2 a_{0}$. The spectral equivalence at all scales is illustrated (similarity of the envelop of the slip distribution).

means that except in the case of extremely large events, with characteristic size much greater than $60 \mathrm{~km}$, the condition $a \gg a_{c}^{\infty}$ is not fulfilled at seismological scales, in contradiction to our initial assumption. Therefore the fault network evolves in the small-scale regime for which the weakening rate of each subfault obeys $\alpha \propto 1 / a$ since $a \ll a_{c}^{\infty}$.

[17] The same reasoning can be applied to the laboratory scale. Typical laboratory experiments lead to $\Delta \tau \simeq 1 \mathrm{MPa}$ and $D_{c} \simeq 10 \mu \mathrm{m}$ leading to $\alpha=\lambda=5 \mathrm{~m}^{-1}$ for the large-scale regime. Therefore the nucleation size (that separates the two asymptotic regimes) is $a_{c}^{\infty} \simeq 0.63 \mathrm{~m}$, a value which is of the order of the size of rock laboratory samples. Therefore, and as for seismological data, the initial assumption $a \gg a_{c}^{\infty}$ is not fulfilled meaning that laboratory samples should also stand in the $\alpha \propto 1 / a$ regime.

[18] Laboratory and seismological studies suggest a scale invariance of the stress drop during a frictional instability
[Beroza and Ellsworth, 1996; Ohnaka and Shen, 1999], leading to a stress drop of the order of $\Delta \tau \simeq 1 \mathrm{MPa}$, even though kilobar fluctuations of stress may occur on a fine scale as individual grains fracture or slide over each other as discussed by Andrews [1981]. Unlike $D_{c}$ which seems to be an effective rather than a physical parameter, the stress drop $\Delta \tau$ appears as a material property (once averaged over lengths much longer than the grain size) related to the strength of the material. Assuming that the stress drop $\Delta \tau$ is constant at all scales, the scaling $\alpha \propto 1 / a$ predicted by our model implies that, under the restricted assumption of a linear weakening law, the slip weakening distance has to obey $D_{c} \propto a$. Our numerical results show that the weakening rate verifies $\alpha=\Delta \tau /\left(\mu D_{c}\right)=\beta_{0}^{*} / a$, with $\beta_{0}^{*} \simeq$ 1.15777388. This equation implies that

$$
D_{c}=\frac{\Delta \tau}{\mu \beta_{0}^{*}} a .
$$




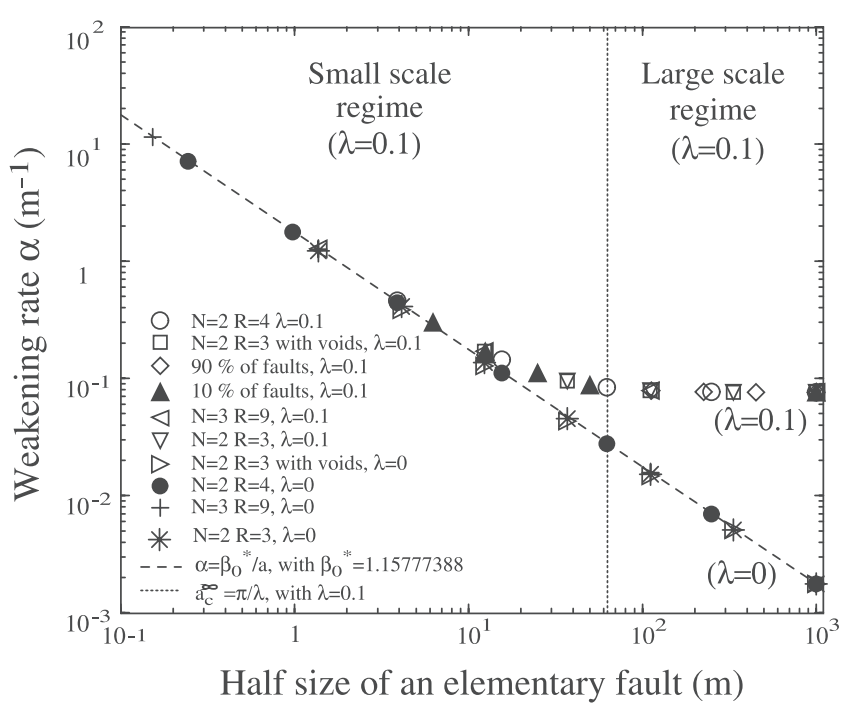

Figure 3. Scaling of various weakening rates $(\lambda=0,0.1)$. The vertical line corresponds to the half nucleation length $a_{c}^{\infty}=\pi / \lambda$, and separates the large-scale and small-scale regimes. At small scales, the weakening rate is well approximated by the asymptote $\alpha=\beta_{0}^{*} / a$, with $\beta_{0}^{*}=$ 1.15777388 .

Taking $\mu=30 \mathrm{GPa}$ and $\Delta \tau \simeq 1 \mathrm{MPa}$ as being representative values of the rigidity and the stress drop, we find that $D_{c} \simeq$ $7.510^{-5} a$. This last estimate is valid only where $a$ represents the typical size of a segment among the fault network, and not the typical size of the contacts between the two surfaces.

[19] Seismological estimates of the characteristic length $D_{c}\left(D_{c} \simeq 1 \mathrm{~m}\right)$ can put an upper bound to the value of $\lambda$. Noting that in the less favorable case, the seismological derivation of $D_{c}$ corresponds to the resolution limit of the inversion, the true value of $D_{c}$ should verify $D_{c} \leq 1 \mathrm{~m}$. Equation (5) can be rewritten using $\alpha=\Delta \tau /\left(\mu D_{c}\right)$ :

$$
D_{c}=\frac{\Delta \tau}{\mu} \min \left[a / \beta_{0}^{*}, 1 / \lambda\right] .
$$

Using $\Delta \tau=1 \mathrm{MPa}, \mu=30 \mathrm{GPa}, \beta_{0}^{*}=1.15777388, a=$ $100 \mathrm{~km}$ as being representative for large earthquakes, we find that $\lambda \leq 7.5 \times 10^{-5}$ such that $D_{c} \leq 1 \mathrm{~m}$. This further justifies why we have only considered fault networks close to the stability boundary $(\lambda \ll 1)$.

[20] From the previous discussion it follows that at all scales (laboratory as well as seismologicafl scale), the weakening rate falls in the regime where $\alpha \propto 1 / a$. This last result may have strong implications on the evaluation of the parameter $D_{c}$ assuming that friction on the fault is a linear law and that the stress drop $\Delta \tau$ is constant at all scales. Under these assumptions, the ratio of $D_{c}^{\text {fault }}$ at the fault scale (with $a^{\text {fault }}=100 \mathrm{~km}$ ) to $D_{c}^{\text {labo }}$ at the laboratory scale (with $\left.a^{\text {labo }}=1 \mathrm{~m}\right)$ is of the order of $D_{c}^{\text {fault }}=\left(a^{\text {fault }} / a^{\text {labo }}\right) D_{c}^{\text {labo }}$. This leads to $D_{c}^{\text {fault }}=1 \mathrm{~m}$ for $D_{c}^{\text {labo }}=10 \mu \mathrm{m}$. Therefore this simple scaling may explain the discrepancy between laboratory and seismological observations. However, this result is based on strong assumptions, i.e., linearity of the weakening law, constant stress drop and barriers of infinite strength between each subfaults. It should be confirmed using more realistic fault models (e.g., three-dimensional fault models with breakable barriers and nonregular fault networks) before any definitive statements can be drawn concerning the scale dependence of the parameter $D_{c}$.

[21] Acknowledgments. We are grateful to C. Dascalu for useful discussion and to J. Schmittbuhl, M. Ohnaka, and C. Péquégnat for their help and advice. This work was supported by ACI "Prévention des catastrophes naturelles." All the computations presented in this paper were performed at the Service Commun de Calcul Intensif de l'Observatoire de Grenoble (SCCI).

\section{References}

Andrews, D. J., A stochastic fault model: 2. Time-dependent case, J. Geophys. Res., 86, 10,821-10,834, 1981.

Beroza, G. C., and W. L. Ellsworth, Properties of the seismic nucleation phase, Tectonophysics, 261, 209-227, 1996.

Campillo, M., and I. R. Ionescu, Initiation of an antiplane shear instability under slip dependent friction, J. Geophys. Res., 102, 20,363-20,371, 1997.

Campillo, M., P. Favreau, I. R. Ionescu, and C. Voisin, On the effective friction law of an heterogeneous fault, J. Geophys. Res., 106, 16,307$16,322,2001$.

Chambon, G., J. Schmittbuhl, and A. Corfdir, Laboratory gouge friction: Seismic-like slip weakening and secondary rate-and state-effects, Geophys. Res. Lett., 29(10), 1366, doi:10.1029/2001GL014467, 2002.

Dascalu, C., I. Ionescu, and M. Campillo, Fault finiteness and initiation of dynamic shear instability, Earth Planet. Sci. Lett., 177, 163-176, 2000. Ide, S., and M. Takeo, Determination of constitutive relations of fault slip based on seismic wave analysis, J. Geophys. Res., 102, 27,379-27,391, 1997.

Korvin, G., Fractal Models in the Earth Sciences, Elsevier Sci., New York, 1992.

Ohnaka, M., Dynamic breakdown processes and the generating mechanism for high frequency elastic radiation during stick-slip instabilities, in Earthquake Source Mechanics, Geophys. Monogr. Ser., vol. 37, edited by S. Das, J. Boatwright, and C. H. Scholz, pp. 13-24, AGU, Washington, D. C., 1986.

Ohnaka, M., and L.-F. Shen, Scaling of the shear rupture process from nucleation to dynamic propagation: Implications of geometric irregularity of the rupturing surfaces, J. Geophys. Res., 104, 817-844, 1999.

Olsen, K. B., R. Madariaga, and R. J. Archuleta, Three-dimensional dynamic simulation of the 1992 Landers earthquake, Science, 278, 834838, 1997.

Scholz, C. H., The Mechanics of Earthquakes and Faulting, Cambridge Univ. Press, New York, 1990.

Voisin, C., I. Ionescu, M. Campillo, R. Hassani, and Q. Nguyen, Process and signature of initiation on a finite fault system: A spectral approach, Geophys. J. Int., 148, 120-131, 2002.

M. Campillo and H. Perfettini, Laboratoire de Géophysique Interne et Tectonophysique, BP 53X, 38041 Grenoble Cedex, France. (Michel. Campillo@obs.ujf-grenoble.fr; Hugo.Perfettini@obs.ujf-grenoble.fr)

I. Ionescu, Laboratoire de Mathématiques, Université de Savoie, Campus Scientifique, 73376 Le Bourget du Lac, France. (ionescu@univ-savoie.fr) 